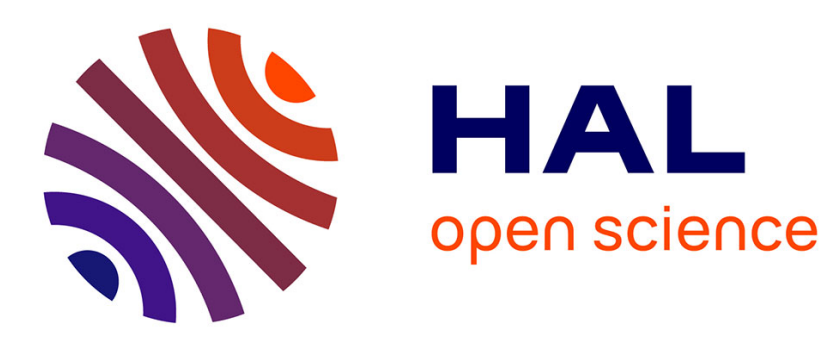

\title{
An Inclined Plane: A Simple Model for the Acoustic Influence of the Flutist's Face
}

Augustin Ernoult, Patricio de La Cuadra, Benoît Fabre

\section{To cite this version:}

Augustin Ernoult, Patricio de La Cuadra, Benoît Fabre. An Inclined Plane: A Simple Model for the Acoustic Influence of the Flutist's Face. Acta Acustica united with Acustica, 2018, 104 (3), pp.496-508. 10.3813/AAA.919192 . hal-01943166

\section{HAL Id: hal-01943166 https://hal.science/hal-01943166}

Submitted on 3 Dec 2018

HAL is a multi-disciplinary open access archive for the deposit and dissemination of scientific research documents, whether they are published or not. The documents may come from teaching and research institutions in France or abroad, or from public or private research centers.
L'archive ouverte pluridisciplinaire HAL, est destinée au dépôt et à la diffusion de documents scientifiques de niveau recherche, publiés ou non, émanant des établissements d'enseignement et de recherche français ou étrangers, des laboratoires publics ou privés. 


\title{
An inclined plane: a simple model for the acoustic influence of the flutist's face.
}

\author{
Augustin Ernoult ${ }^{1,3)}$, Patricio de la Cuadra ${ }^{2)}$, Benoît Fabre ${ }^{3)}$ \\ 1) Laboratoire de Mécanique et d'Acoustique CNRS - UMR 7031 \\ 4 impasse Nikola Tesla CS 40006, 13453 Marseille Cedex 13, France \\ 2) CITA, Pontificia Universidad Católica de Chile \\ 3) LAM, Institut Jean le Rond d'Alembert, UMR 7190, CNRS, \\ UPMC Univ Paris 06, Sorbonne Universités, F-75005 Paris, France
}

\begin{abstract}
Summary
In some flute-like instruments, the musician places their mouth near an open-end of the pipe, which modifies the radiation of the pipe and its acoustic resonances. To improve the prediction of this modification, this work presents a study on the effect of an inclined plane at the end of a radiating pipe. The radiation impedance is first measured for different positions of the plane. After verifying that the plane mainly adds an inertance to the radiation, a new simpler and more convenient measurement method is proposed. The behavior observed in these measurements is generalized with the use of finite element simulations. Finally, a global low-frequency model of the effect of an inclined plane on both the imaginary and the real part of the radiation impedance is proposed. This model is consistent with the well-established radiation impedances for unflanged and flanged pipes.
\end{abstract}

\section{Introduction}

\subsection{The flutist: a bore profile element}

Flute-like instruments are musical instruments in which the sound is created by a jet-edge interaction coupled with an acoustic resonator [1]. The jet is formed by applying a supply pressure upstream from a channel, the outlet of which is directed towards the edge.

The channel is formed either between the lips of the musician (as in transverse and notch flutes), or by the instrument maker (as in recorders and organ pipes). All these instruments share the feature of having a specific opening at the end of the resonator where the sound is generated. This opening is called the window and is located between the outlet of the channel and the edge or labium. The jet-edge interaction induces a fluctuating pressure difference acting as a sound source.

The frequency of the sound generated by these instruments depends on the balance between the phase induced by the resonator and the delay produced by the convection of a perturbation along the jet [2]. The frequency response of the instrument depends on the window impedance $[1,3]$. In recorder-like instruments, this impedance is fixed by the instrument maker. It can be estimated from the window geometry [4].

However, in transverse and notch flutes, the player's face is placed near this opening to blow on the edge. While playing, the player adjusts the position of his/her face to control the pitch of the note played [5, 6, 7, 8, 9, 10]. The window impedance is therefore continuously modified by the player.

The acoustic resonances of the instrument in the absence of a musician are different from the expected sounding frequencies, and this detuning is different for each fingering. This results in a passive tuning profile different for every flute depending on the model, the instrument maker, the historical period, etc. [11, 7, 12, 13, 14]. This profile influences the quality and the playability of the instrument.

The lack of information regarding the acoustic effect of the presence of the player's face limits the possibility of applying traditional resonant frequency calculations based on 1D acoustic theory to this kind of instrument. For example, the calculation of Wolfe [11] uses a fixed length correction to take into account the player's lips and face, which does not allow interpreting the tuning profile of an existing instrument. It is therefore impossible to predict the sounding frequency of a flute from its geometry alone. The study of pitch accuracy, the quality, and the playability of an instrument can therefore only be studied in comparison to a reference instrument [12]. 
Furthermore, instrument makers can not even use acoustic tools such as optimization algorithms to create new instruments. Indeed, a reference tuning profile is necessary and this profile can not be based on acoustic criteria.

The work presented here gives some tools to better understand the musician gesture and how the resulting geometry relates to the acoustic behavior of the musician and instrument ensemble. The overall objective is to describe how the musician's face modifies the resonances of the instrument as function of its position.

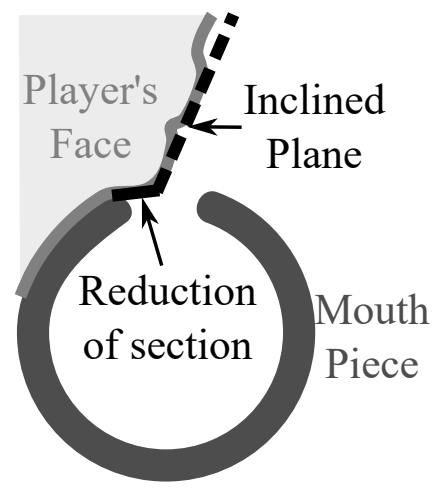

Figure 1: Sketch of a musician's lips in front of the mouth opening

The geometrical modification of the window by the musician's face can be divided into two principal elements: a reduction of the outside area $S_{o}$ of the opening and the presence of an inclined plane in front of the opening formed by the lips and the musician's face (Fig. 1). The former has an effect on the radiation that is already well known. The imaginary part of the radiation impedance is generally modeled by an inertance $m_{r}$, similar to a length correction $l_{r}$ at low frequency, which in turn is proportional to the equivalent radius of the opening section $r_{o}=\sqrt{\frac{S_{o}}{\pi}}[15,16,17,18,19,20]$ :

$$
m_{r}=l_{r} \frac{\rho}{S_{o}}=\delta \frac{\rho}{S_{o}} r_{o}
$$

with $\rho$ the density of the air and $0.613<\delta<0.824$ a dimensionless coefficient which depends on the wall thickness $(\delta=0.824$ for an infinite flanged pipe and $\delta=0.613$ for an unflanged pipe with infinite thin wall). Thus, a reduction of the cross-sectional area decreases this length correction.

The presence of an inclined plane induces a modification of the radiation impedance that, to our knowledge, remains undocumented. The main goal of this work is to study and to model how an inclined plane modifies the radiation impedance of an opening.

After revisiting previous studies on radiation impedance, the effect of the plane is studied experimentally by two complementary methods. The first method involves measuring the impedance of a pipe with a plane placed in front of an opening at varying angles. In the second method, this pipe is used as the resonator of a self-sustained sound source and the modification of the impedance by the presence of the inclined plane is inferred from the variation of the sounding frequency. These two sets of measurements lead to the proposition of an empirical model with coefficients that are related to the geometry of the opening. Finite element simulations are then used to simulate different openings. Finally, a comprehensive empirical model of the effect on the radiation impedance of a wide inclined plane in front of a radiating aperture is proposed.

\subsection{Simplification and Generalization}

The radiation impedance of openings with different geometries has already been studied; e.g. the open ending of pipe with different flanges $[15,18,20]$ or the radiation of side hole $[16,17,19]$. All the radiation impedances, defined here as the ratio of the complex amplitudes of acoustic pressure $\left(P_{a c}(k)\right)$ and mean acoustic velocity $\left(V_{a c}(k)\right)$ at the end of the pipe, can be written using the same global formulation:

$$
Z_{r}=\frac{P_{a c}(k)}{V_{a c}(k)}=j m_{r} c k+\zeta \frac{\rho c}{2 \pi} k^{2}
$$

with $c$ the sound velocity, $\rho$ the air density, $k$ the wavenumber, and $m_{r}$ and $\zeta$ two real frequency-dependent coefficients related to the opening geometry. The inertance, $m_{r}$, influences the resonant frequencies of the pipe, and has therefore been studied more than the dimensionless coefficient $\zeta$ which influences the quality factors 
and the amplitudes of the resonances. These two coefficients are approximately constant at low frequencies. The two most analyzed geometries are the radiation of an unflanged pipe with thin wall for which [15]:

$$
\left\{\begin{array}{l}
m_{r}^{(\text {unflg })}=\frac{\rho}{\pi r_{o}} 0.613 \\
\zeta^{(\text {unflg })}=\frac{1}{2}
\end{array}\right.
$$

and the infinite flanged pipe for which $[18,20]$ :

$$
\left\{\begin{array}{l}
m_{r}^{(f l g)}=\frac{\rho}{\pi r_{o}} 0.824 \\
\zeta^{(f l g)}=1
\end{array}\right.
$$

where $r_{o}=\sqrt{S_{o} / \pi}$ is the equivalent radius of the opening.

Radiation impedance for configurations closer to the one studied here, can be found in the literature. The effect of a parallel plane in front of an opening has been studied by Dalmont et al. [18] for the purpose of modeling the effect of the key suspended above a tone hole. In this case the plane is not inclined (Fig. 2,a)). The effect of the plane is interpreted by Dalmont as a correction $\Delta m_{r}$ of the radiation inertance without plane $m_{r 0}$ (unflanged pipe). If the plane distance $h_{p}$ is short in comparison with the radius opening $r_{o}\left(h_{p} \ll r_{o}\right)$, the inertance correction is approximately proportional to the inverse of this distance:

$$
\Delta m_{r}=m_{r}-m_{r 0} \underset{h_{p} / r_{o} \rightarrow 0}{\approx} \frac{\rho}{\pi} \frac{1}{3.5 h_{p}}
$$

a)

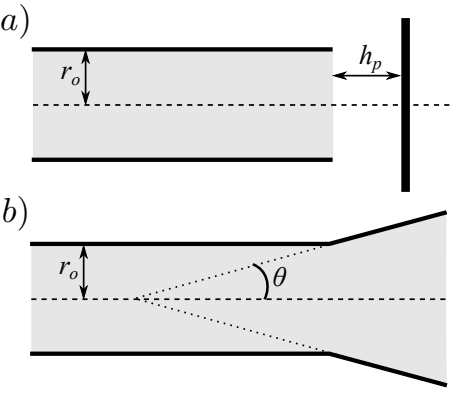

Figure 2: a): Sketch of the parallel plane in front of the opening studied by Dalmont et al. [18]. b):Sketch of the junction of a cylinder and truncated cone studied by Kergomard et al. [21].

The junction of a cylinder and a truncated cone, studied by Kergomard et al. [21], is also comparable to the problem studied here. Indeed, the case where the truncated cone is semi-infinite is equivalent to a cylinder surrounded by an inclined wall (Fig. 2,b)). In this case, for low frequencies, the output impedance of the cylinder radiating into the cone $Z_{r}^{(\text {cone })}$ is approximately:

$$
Z_{r}^{(\text {cone })} \approx j k c \frac{M+\xi V}{1+j k c M / \xi}
$$

The inertance $M$ and the two coefficients $V$ and $\xi$ are dependent on the half-angle of the truncated cone $\theta$ and the radius of the cylinder $r_{o}$ :

$$
\left\{\begin{aligned}
M & =\frac{\rho}{2 \pi r_{o}} \cot (\theta / 2) \\
\xi & =\frac{\rho}{c \pi r_{o}^{2}} \cos ^{2}(\theta / 2) \\
V & =c r_{o} \sin (\theta) \frac{2+\cos (\theta)}{3(1+\cos (\theta))^{2}} .
\end{aligned}\right.
$$

This expression ensures the transition between plane waves into the cylinder and spherical waves into the cone. It is only valid for acute cones $(\theta \leq \pi)$.If the angle of the cone is sufficiently wide, this expression can be approximated for low frequencies by the expression of Eq.(2), leading to an inertance $m_{r}^{(c o n e)}$ and a coefficient $\zeta^{(\text {cone })}[21]$ :

$$
\left\{\begin{aligned}
m_{r}^{(\text {cone })} & =M+\xi V \\
\zeta^{(\text {cone })} & =\frac{2 \pi}{\rho c} \frac{M^{2}}{\xi}=\frac{1}{1-\cos (\theta)} .
\end{aligned}\right.
$$

However, for small angles $(\theta \ll 1)$, the truncated cone starts to resemble a cylinder. The inertance $m_{r}^{(c o n e)}$ diverges and the development of Eq.(6) gives $Z_{r}^{(\text {cone })} \approx \xi$, which corresponds to the characteristic impedance 
of the cylinder. The output impedance is thus independent of the frequency $\left(m_{r}^{(\text {cone })}=0\right.$ and $\left.\zeta^{(\text {cone })}=0\right)$. Therefore, it is impossible to use this model to generate general expressions of the inertance and coefficient $\zeta$ that are valid for all angles of the truncated cone. The impossibility of generalization is due to the truncation of the cone. Indeed, for small angles, the volume truncated tends towards infinity.

The similarity with our geometry suggests that the radiation impedance of an opening with an inclined plane could be correctly described by Eq.(2) with an inertance $m_{r}$ and a coefficient $\zeta$ that are independent of frequency in the low-frequency approximation. Furthermore, the effect of the inclined plane on the inertance could be expressed as a modification $m_{\beta}$ of the inertance of an unflanged pipe $m_{r 0}$ :

$$
m_{r}=m_{\beta}+m_{r 0} .
$$

Finally, under these assumptions, the aim of this study is to propose expressions for the inertance $m_{\beta}$ and for coefficient $\zeta$, accounting for the position of the inclined plane and the geometry of the opening. For this first study, only circular planes of much greater area than the opening section $\left(S_{\text {plane }} \gg S_{o}\right)$ are considered. Two methods are proposed to measure these parameters.

\section{Passive resonance measurement}

\section{$2.1 \quad$ Measurement principles}

The protocol used here is similar to the one used in a previous study of the radiation impedance of the window of recorder-like instruments [4]. The input impedance $Z_{\text {in }}$ of a finite open pipe is measured by an impedance sensor (Fig. 3). The radiation impedance of the opening is then estimated through the transfer matrix propagation of the sound inside the pipe. This measurement is made with and without an inclined plane in front of the opening for different plane radii and different tilt angles.

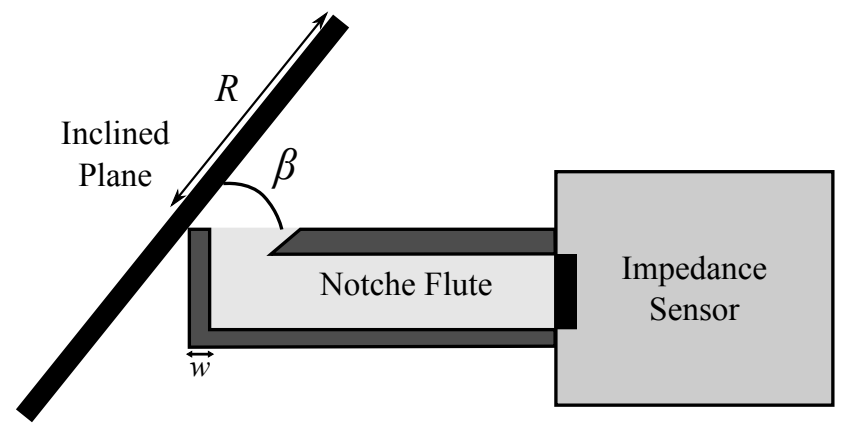

Figure 3: Sketch of the set-up used for passive measurements.

The pipe used for these measurements is the cylindrical upper part of a 3D printed plastic notch flute (Quena) of internal radius $r_{p}=9.5 \mathrm{~mm}$ and length $L_{p}=128 \mathrm{~mm}$. The notch is the hole where the player places his/her face. This hole is at one boundary of the resonator, which enables the placement of the inclined plane (Fig. 3), in contrast to a transverse flute where this hole is located in the middle of the mouthpiece.

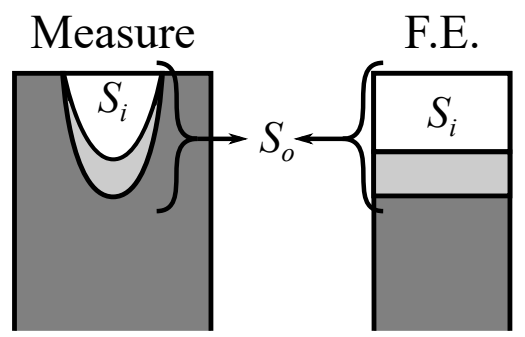

Figure 4: a) Sketch of the window used for the measurements. b) Equivalent model of the window used in the finite element simulations (Sec. 4).

The notch used here is a parabolic hole. Due to the presence of the edge (Fig. 3), the external opening area $S_{o}=1.32 \mathrm{~cm}^{2}$ of the hole is larger than the inside opening area $S_{i}=0.93 \mathrm{~cm}^{2}$ (Fig. 4, a). The planes used to simulate the player's face are circular planes made of plastic ( $4 \mathrm{~mm}$ thickness) with different radii 
$(R=4 \mathrm{~cm}, 8 \mathrm{~cm}, 12 \mathrm{~cm})$, ensuring that $S_{\text {plane }} \gg S_{o}$. The plane is centered at the extremity of the notch and inclined to the notch plane with different values of the tilt angle $\beta$ ranging from $\pi / 36$ to $\pi / 2$ (Fig. 3).

The impedance sensor used was developed by Le Roux [22]. For each measurement, responses from five sweeps in the range $100 \mathrm{~Hz}$ to $3000 \mathrm{~Hz}$ of five seconds each are averaged. This procedure, including the placement of the inclined plane, is repeated three times for each value of the pair of parameters $(R, \beta)$, giving three measurements of $Z_{\text {in }}$ per configuration.

In the low frequency assumption of plane waves $\left(k r_{p} \ll 1.8\right)$, the radiation impedance $Z_{r}$ can be obtained from the input impedance $Z_{i n}$, using the transfer matrix of a cylinder, with the formula [23, 24]:

$$
Z_{r}=\frac{Z_{c} \tanh \left(\Gamma L_{p}\right)-Z_{i n}}{\frac{Z_{i n}}{Z_{c}} \tanh \left(\Gamma L_{p}\right)-1},
$$

where $\Gamma$ is the complex propagation coefficient and $Z_{c}$ the characteristic impedance of the cylinder. Both account for the visco-thermal losses related to the cross-sectional area of the tube and the wavenumber [25]:

$$
\left\{\begin{array}{c}
\Gamma=j k \phi_{\Gamma} \\
Z_{c}=\frac{\rho c}{S_{p}} \phi_{Z_{c}}
\end{array},\right.
$$

with $\phi_{\Gamma}$ and $\phi_{Z_{c}}$ two dimensionless functions [25] introducing corrections due to viscous and thermal effects on the propagation. The two dimensionless functions depend on the Stokes number similar to a dimensionless radius: $r_{v}=r_{p} \sqrt{\omega \rho / \eta}$, where $\eta$ is the shear viscosity coefficient. The values of the density $\rho$, the speed of sound $c$, and the shear viscosity $\eta$ are estimated from the temperature [25]. For large values of the Stokes number, a second order development in $1 / r_{v}$ gives [24]:

$$
\left\{\begin{array}{l}
\phi_{\Gamma}=1+\frac{\alpha_{1} \sqrt{-2 j}}{r_{v}}-j \frac{\alpha_{2}}{r_{v}^{2}} \\
\phi_{Z_{c}}=1+\frac{A_{1}}{r_{v}}+\frac{A_{2}}{r_{v}^{2}}
\end{array},\right.
$$

where $\alpha_{1}, \alpha_{2}, A_{1}, A_{2}$ are coefficients that depend on the Prandtl number $P_{r}$ and on the heat capacity ratio $\gamma$. For air, these two parameters are assumed to be constant $\left(P_{r}=0.71\right.$ and $\left.\gamma=1.402\right)$ which gives: $\alpha_{1}=1.044$; $\alpha_{2}=1.080 ; A_{1}=0.370$, and $A_{2}=1.147$ [24].

This calculation is first applied to measurements of the input impedance of the flute mouthpiece with the notch blocked (closed pipe) to determine precisely the length of the pipe. Then it is used to extract the radiation impedance $Z_{r}$ for each pair of inclined plane parameters $(R, \beta)$. Assuming that this impedance follows the expression of Eq.(2), the real part of the radiation impedance is normalized by $\rho c k^{2} /(2 \pi)$ and the imaginary part by $\rho c k$ (the normalization by $\rho$ reduces the temperature effect on the measurement).

\section{$2.2 \quad$ Influence of the plane inclination}

The imaginary and real parts of the radiation impedance obtained with one given plane $(R=8 \mathrm{~cm})$ and different tilt angles are compared in the frequency domain to examine the effect of the plane (Fig. 5). As the plane is increasingly inclined (smaller $\beta$ ), there is a greater modification to the real and imaginary components of the impedance (Fig. 5).

Due to the normalization of the imaginary and real components by $k$ and $k^{2}$, respectively, the curves are expected to be constant at low frequency. But inaccuracies in the measurements induce frequency dependent variations which cause the data to deviate from this expected behavior (Fig. 5). The three repetitions of the measurements (Sec. 2.1) superposed in Figure 5, demonstrate a good repeatability of the measurements for both the imaginary and the real components. This repeatability includes the frequency dependent variations. The deviation between the measurements with the same tilt angle are within $1 \%$ for the imaginary component and within $5 \%$ for the real component. The frequency dependent variations have a similar pattern in all the measurements and exist both in the presence and in the absence of the plane. It seems inherent to the measurements method. As presented in Section 2.1, the radiation impedances $Z_{r}$ are not measured directly. They are estimated from the input impedance of the notch flute $Z_{i n}$ through a model of the acoustic propagation into the pipe (Eq. 10). The observed impedance $Z_{r}$ is small compared to the measured impedance $Z_{i n}$. The inaccuracies in the measurements (mainly due to low signal-to-noise ratio at the anti-resonances and to sensor artifacts) and in the model of the propagation into the pipe (the geometric parameters of the tube and the visco-thermal losses used in Eq. 10) have a non-negligible effect on the estimated impedance. Together, these inaccuracies induce the frequency dependent variations.

The normalized imaginary component shows limited dependence on frequency, especially for low frequencies $\left(k r_{p}<0.25\right)$ (Fig. 5,a). In spite of the frequency dependent variations discussed previously, the normalized 

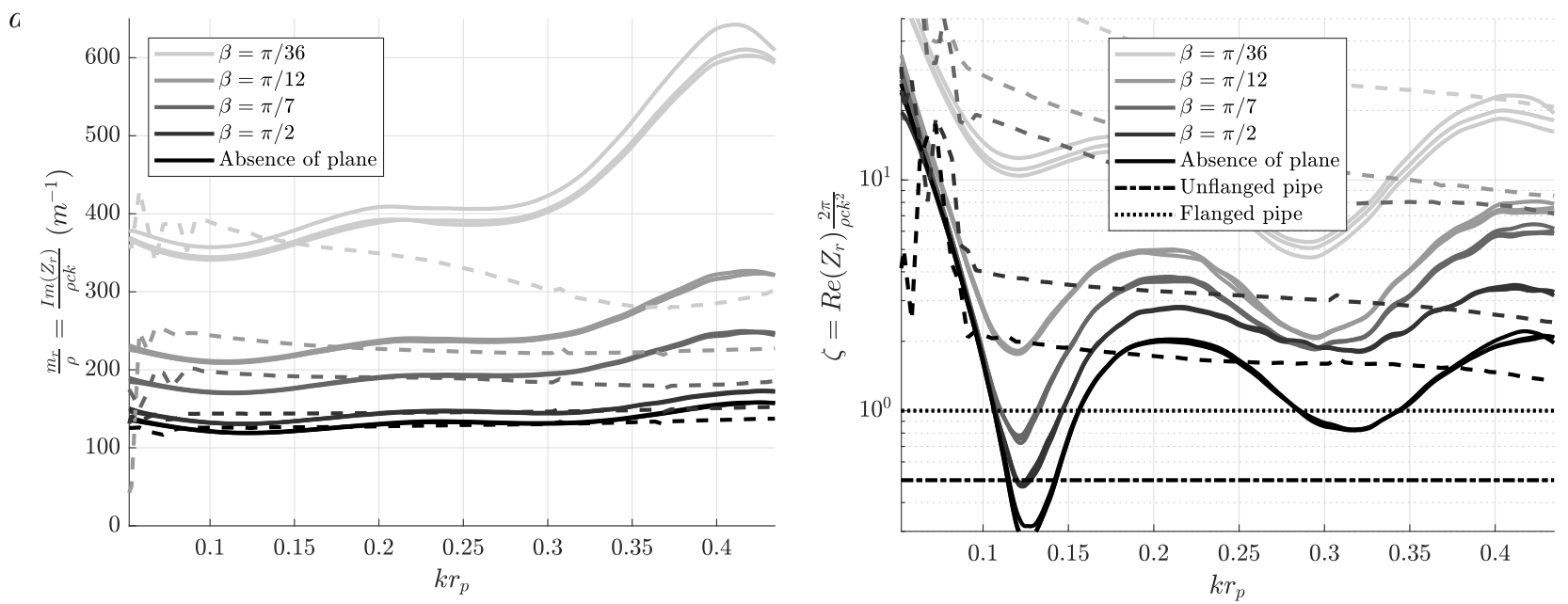

Figure 5: Radiation impedance ( $a$ : imaginary part; $b$ : real part), with an inclined plane $(R=8 \mathrm{~cm})$ and different values of the tilt angle $(\pi / 36<\beta<\pi / 2)$ indicated by the grey scale. The wavenumber $k$ is normalized relatively to the pipe radius $r_{p}$ (i.e. $\left.k r_{p}\right)(f \in[300 \mathrm{~Hz}, 2500 \mathrm{~Hz}])$. Passive measurements (solid lines); FE simulations from Sec. 4 (dashed lines); the theoretical real parts of flanged and unflanged pipe (dotted and dashed-dotted lines).

impedances are constant at low frequencies $\left(k r_{p}<0.25\right)$ within $5 \%$ percents of their mean values for each angle $\beta$. These measurements are consistent with the assumption that, at low frequencies, the plane adds a constant inertance $m_{\beta}$ to the radiation impedance. The smaller the angle $\beta$, the bigger this inertance. It is possible to quantify this inertance $m_{\beta}$ for each plane configuration by considering the average value of the normalized imaginary component at low frequencies $\left(k r_{p}<0.25\right)$ (Fig. 5, a). These values are discussed in Section 2.4.

It is more difficult to validate the assumptions as they apply to the real component, because the real part of the radiation impedance is small compared to the imaginary part for low frequencies (Eq. (2)). Therefore, the small inaccuracies discussed previously have a bigger effect (Fig. 5, $b$ ). Even assuming that the coefficient $\zeta$ is constant in the low frequency range and that the frequency dependent variations observed are measurements artifacts, it is difficult to estimate the value of this coefficient. Only a qualitative observation can be made: the smaller the tilt angle $\beta$, the larger the coefficient $\zeta$.

\subsection{Influence of the plane radius}

a)

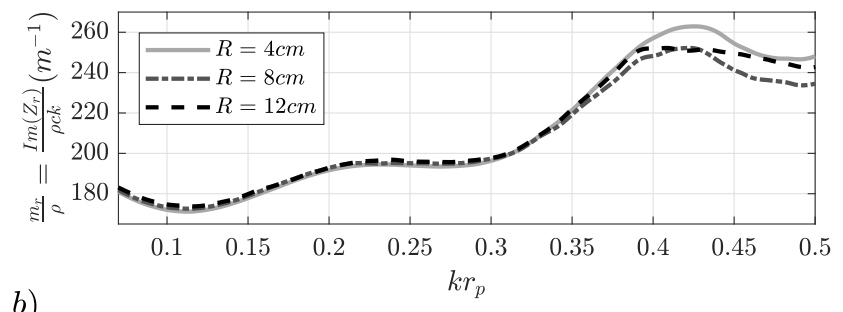

b)

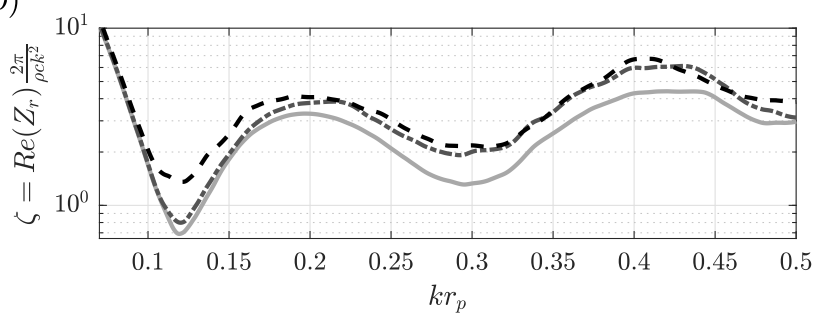

Figure 6: Radiation impedance with planes at similar inclination $(\beta=\pi / 7)$ but different radii $(R=4 \mathrm{~cm}$, $R=8 \mathrm{~cm}$ and $R=14 \mathrm{~cm})$ compared to a reference $\left(R_{r e f}=8 \mathrm{~cm}\right)(a)$ : imaginary part, $\left.b\right)$ : real part $)$. The wave length $\lambda$ is normalized by the reference radius. 
The influence of the radius of the circular plane is now investigated. Three planes with different radii are tested $(R=4 \mathrm{~cm} ; R=8 \mathrm{~cm} ; R=12 \mathrm{~cm})$. The data measured for $\beta=\pi / 7$ with these three planes are superposed in Figure 6 . The radius of the plane has a smaller effect than its inclination both on the imaginary and the real components (Fig.5).

The imaginary components are similar (Fig. 6,a). The deviation at a given frequency is within $1 \%$ at low frequencies $\left(k r_{p}<0.25\right)$ and stays within $6 \%$ at higher frequencies. To a first approximation, the imaginary component of the radiation impedance, and therefore the inertance $m_{\beta}$, are assumed independent of the plane size. It is important to note that the wavelengths studied stay greater than the radii of the planes $(\lambda \in$ $[11.5 \mathrm{~cm}, 115 \mathrm{~cm}])$.

It is less obvious how to interpret the observations on the real components (Fig. 6, $b$ ). The real component obtained with $R=4 \mathrm{~cm}$ is below the others over the entire frequency range. It induces a mean deviation of $30 \%$. The same type of variation is also observed at low frequencies between the two largest planes (Fig. 6, $b$, for $\left.k r_{p}<0.2\right)$. However, this effect is small compared with the frequency dependent variations caused by the measurement artifacts discussed previously (Sec. 2.2). The size of the plane seems to have an effect on the real component of the radiation impedance, but the measurements presented here can not be used to quantify it. For the wide planes $(R=8 \mathrm{~cm}$ and $R=12 \mathrm{~cm})$ the influence of size is small compared to the one of the tilt angle (Fig.5).

As a first approximation, the radius of the inclined plane is assumed to have negligible influence on the radiation impedance. Only planes of greater surface area than the opening area are used here $\left(S_{\text {plane }}>S_{o}\right)$. Under this condition, the planes are large enough to be considered infinite.

\subsection{Angle dependence of the inertance}

For each value of the tilt angle, nine radiation impedance measurements are performed ( 3 for each of the 3 planes). For each measurement, a value of the total radiation inertance $m_{r}$ is estimated by taking the average value of the normalized imaginary part in low frequency $\left(f \in[300 \mathrm{~Hz}, 1500 \mathrm{~Hz}]\right.$ or $\left.k r_{p} \in[0.05,0.25]\right)$. The part $m_{\beta}$ of this inertance due to the inclined plane is estimated by taking the difference between the inertance $m_{r}$ and the inertance $m_{r 0}$ measured in the absence of a plane (Eq.(9)). The inertances $m_{\beta}$ obtained are normalized by the air density $\rho$ to compensate variations due to the temperature. The mean of these values give an estimation of the inertance $m_{\beta}$ for each value of the tilt angle $\beta$ (Fig. 7). Due to the good repeatability of the measurements (Sec. 2.2, Fig. 6), the inaccuracy of the inertance $m_{\beta}$ is dominated by the frequency dependent variations discussed in Sec. 2.2 (around 5\%) and not by the variation between measurements ( $\mathbf{i} 1 \%$ ). The standard deviation of the imaginary component over the low frequency range is used to quantify the accuracy of the estimation of the inertance $m_{\beta}$ (error-bars in Fig. 7).

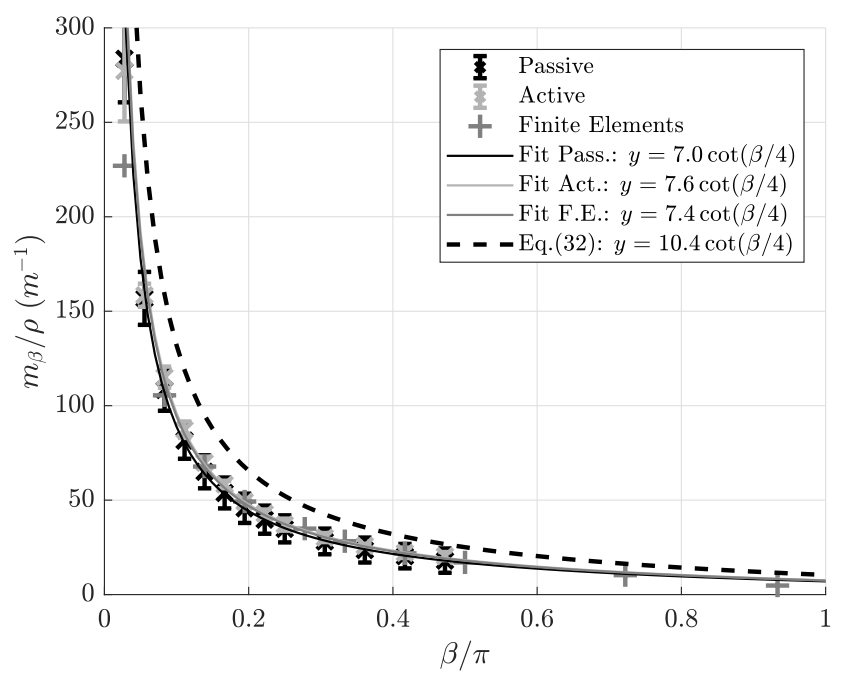

Figure 7: Inertance $m_{\beta}$ normalized by the air density $\rho$ as function of the angle $\beta$. The error bars correspond to the standard deviation of the normalized imaginary component over the frequency range $\left(k r_{p}<0.25\right)$ for each angle. Passive measurements (black x markers) and fitted model (black line); FE simulations (dark gray + markers) and fitted model (dark gray line); active measurements (pale gray x markers) and fitted model (pale gray line); Model of Eq.(32) (dashed black line).

Two asymptotic behaviors are observed. For small angles the inertance tends towards infinity which corre- 
sponds to the impedance of a closed end:

$$
\lim _{\beta \rightarrow 0} m_{\beta}=+\infty
$$

For large angles, the extra inertance $m_{\beta}$ tends to zero:

$$
\lim _{\beta \rightarrow \beta_{0}} m_{\beta}=0
$$

where $\beta_{0}$ is the limit angle for which the effect of the plane is null. This angle $\beta_{0}$ can be interpreted as the angle for which the radiation is no longer affected by the presence of the plane. The function $\cot (\beta / B)$ follows the asymptotic behavior in the data. The coefficient $B$ is a dimensionless coefficient. Following Eq.(14), it is linked to the limit angle by $\beta_{0}=B \pi / 2$.

The behavior of this function is sensitive to the coefficient $B$ only for large angles $\left(\beta\right.$ close to $\left.\beta_{0}\right)$, and it is difficult to estimate the numerical value of $B$ from the measurements. However, the value of the limit angle is limited by the range $\pi<\beta_{0} \leq 2 \pi$. Indeed, when $\beta=\pi$, the configuration is similar to a flanged pipe for which the presence of the plane (or the flange) affects the radiation. The value $B=4\left(\beta_{0}=4 \pi / 2\right)$ allows good modeling of both the inertance and the real part of the impedance (Fig. 7 and Sec. 4.3). At this value, the angle $\beta_{0}$ for which the plane has no effect equals $2 \pi$. For this angle, the plane is placed along the pipe wall and the hole radiates like an unflanged pipe (Fig. 3).

The inertance $m_{\beta}$ behaves like the function:

$$
m_{\beta}=C \cot (\beta / 4),
$$

where $C$ is a coefficient with the dimension of an inertance. The total radiation inertance is therefore:

$$
m_{r}=C \cot (\beta / 4)+m_{r 0}
$$

The value of the constant $C$ is obtained by minimizing the function:

$$
g(C)=\sum_{\beta} \frac{\left|C \cot (\beta / 4)+\epsilon_{0}-\overline{m_{\beta}}\right|}{\operatorname{std}\left(m_{\beta}\right)},
$$

where $\overline{m_{\beta}}$ and $\operatorname{std}\left(m_{\beta}\right)$ are the mean value and the inaccuracy of the measured inertance for each value of $\beta$, respectively. Including the coefficient $\epsilon_{0}$ in the optimization process accounts for a possible systematic error in the estimation of the inertance $m_{r 0}$ in the absence of a plane. The value ${ }^{1}$ of this coefficient $\left(\epsilon_{0} \approx-10 \mathrm{~kg} \cdot \mathrm{m}^{-4}\right)$ is subtracted from the data presented (Fig. 7).

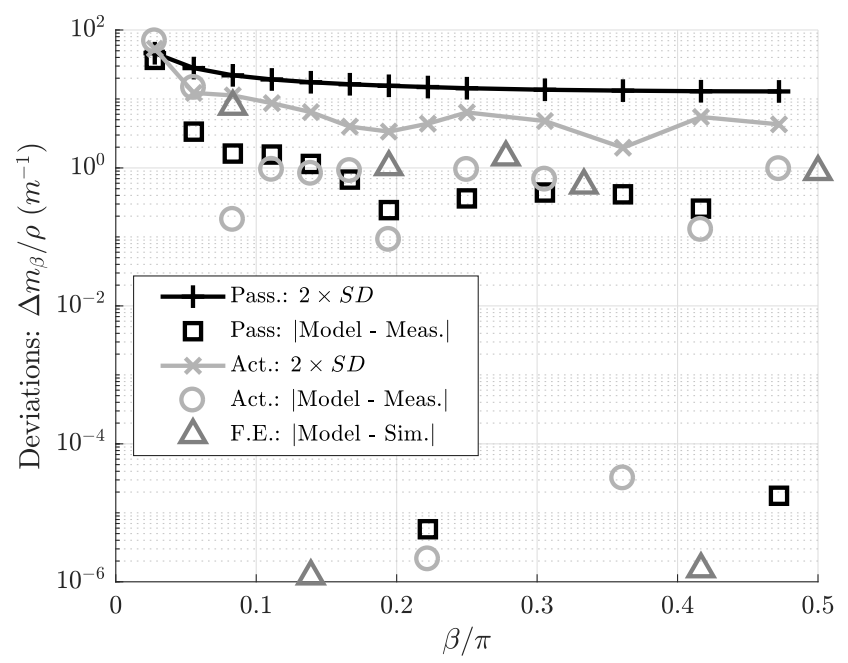

Figure 8: Comparison between the standard deviations (SD) of the measurements and the difference between the values of $m_{\beta}$ predicted by the fitted models and the ones estimated from the measurements or the simulation.

\footnotetext{
${ }^{1}$ The value of $\epsilon_{0}$ is the same order of magnitude as the standard deviation of $m_{r 0}$. Here, $\left|\epsilon_{0}\right| / \overline{m_{r 0}} \approx 6 \%$, and std $\left(m_{r 0}\right) / \overline{m_{r 0}} \approx$ $4.5 \%$. This bias may be due to the frequency dependent variations on the measurements (Fig. $5, a$ ). It could be also due to the width $w$ of the boundary wall on which the plane is placed $(w \approx 3 m m$, Fig. 3$)$. Even in the absence of a plane, this wall slightly limits the acoustic field, similar to a small plane inclined of $\beta=\pi$.
} 
After optimization of the coefficient $C$, the fitted model passes through all the error-bars (Fig. 7). For each angle $\beta$, the difference between the value of $m_{\beta}$ predicted by the fitted model and the one estimated from the measurement is lower than the uncertainty of measurement, which, in the current work, is defined as two times the standard deviation (Fig. 8). It validates the choice of the coefficient $B=4$ in the model.

Before examining how the coefficient $C$ depends on the geometry of the aperture, a second method is proposed to verify the behaviour of the modified inertance.

\section{Active behavior measurements}

\subsection{Measurement principles}

Assuming that the inclined plane modifies the imaginary part of the radiation impedance by adding an inertance without frequency dependence at low frequencies, it is not necessary to measure this effect over a large range of frequencies. The inertance can be estimated from a measurement at only one frequency, making the measurement faster and simpler.

For a self-sustained instrument supplied by a steady source of energy, the sounding frequency is shifted when the resonator is modified. Assuming that the type of modification is known (here an added inertance), it is possible to quantify this modification from the measurement of the sounding frequency [26].

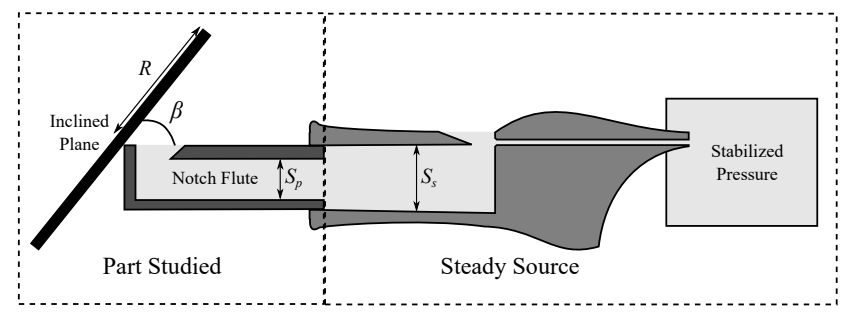

Figure 9: Sketch of the setup used for passive measurements.

For this measurement, the impedance sensor is replaced by a mouthpiece of a tenor recorder: a cylindrical pipe with an internal cross-sectional area $S_{s}=3.9 \mathrm{~cm}^{2}$ and a length $L_{s}=127.7 \mathrm{~mm}$. This sound source is supplied with a steady flow ensured by a flow controller (smart mass flow BROOKS, model 5851S) from a high pressure air supply (Fig. 9). A pressure sensor (Endevco $8507 \mathrm{C}$ ) is placed into the pressure reservoir to control the stability of the supply, and a microphone (B\&K 4938) is placed through the wall of the recorder to measure the internal acoustic pressure. All these elements constitute the self-sustained sound source, and the notch flute studied previously with or without the inclined plane is part of the resonator. For each position of the plane, the internal acoustic pressure is recorded for $5 \mathrm{~s}$ with a sampling frequency $f_{e}=51.2 \mathrm{kHz}$ and the fundamental frequency is estimated. The sounding frequency varies between $f_{w o}=473.6 \mathrm{~Hz}$ without a plane and $f=418.8 \mathrm{~Hz}$ for a tilt angle $\beta=\pi / 36$.

At low frequencies, the inertance $m_{\beta}=m_{r}-m_{r 0}$ added by the inclined plane has a similar effect on the sounding frequency as a modification $l_{\beta}$ of the resonator length, with $l_{\beta}=m_{\beta} S_{s} / \rho$ for $k l_{\beta}<1$. It is therefore possible to estimate the inertance added by the plane by comparing the sounding frequency without plane $f_{0}$ and the sounding frequency with the plane $f$ :

$$
m_{\beta}=l_{\beta} \frac{\rho}{S_{s}}=\left(1-A^{2}\right) \frac{\rho}{S_{s}}\left(\frac{c}{2 f}-\frac{c}{2 f_{0}}\right) .
$$

The coefficient $A$ appears if the internal section $S_{s}$ of the recorder mouth piece is different from the one of the notch flute head $S_{p}$ (App. A):

$$
A \approx j\left(\frac{S_{p}}{S_{s}}\right) \tan \left(k L_{p}\right)
$$

with $L_{p}$ the length of the notch flute head (Sec. 2.1). This coefficient allows the same sound source to be used with pipes of different internal radius. This method measures changes of the radiation inertance, $m_{\beta}=m_{r}-m_{r 0}$, but it can not provide an absolute value of the radiation inertance $m_{r}$. For this method, the measurement is again performed three times for each plane radius and tilt angle $\beta$. 


\subsection{Results}

As with the first method presented, the size of the plane does not strongly influence the inertance measured. For each inclination of the plane, the nine measurements of the inertance difference $m_{\beta}=m_{r}-m_{r 0}(3$ for each size) are grouped allowing the estimation of a mean value and the repeatability of the measurements (Fig. 7 ). While the impedance measurements described in the previous section (Sec. 2.1) correspond to the low acoustic level linear regime, the second method can be used to validate the results of the imaginary part of the radiation impedance at standard playing level.

A model similar to the one proposed for the first method (Eq.(16)) is fitted to this data. The values of coefficients $C$ and $\epsilon_{0}$ are obtained by optimization (Eq.(17)): $C / \rho \approx 7.6 \mathrm{~m}^{-1}$ and $\epsilon_{0} \approx-11 \mathrm{~kg} \cdot \mathrm{m}^{-4}$. The value of $\epsilon_{0}$ is close to that obtained using the first method (Sec. 2.4).

The values of the inertance $m_{\beta}$ and the coefficient $C$ of the fitted model are similar to the ones obtained with the first method (Fig. 7), which validates the method proposed here. The difference between the model and measurements are, again, lower than the standard deviation of the measurements (Fig. 8).

The coefficient $C$ depends on the opening geometry. Instead of manufacturing several mouthpieces to investigate this dependence, a numerical simulation approach is chosen.

\section{Simulation of the radiation}

To study the dependence of the coefficient $C$ on the geometry of the opening, several geometries are simulated using Finite Element methods (F.E.) in which the 3D Helmholtz equation is solved. In this simulation, the geometry and the wave propagation are well controlled. It is therefore possible to study both the imaginary and the real part of the radiation impedance. The numerical simulation is validated by comparing the simulated radiation impedance with the measurements for each position of the inclined plane.

\subsection{Simulation settings}

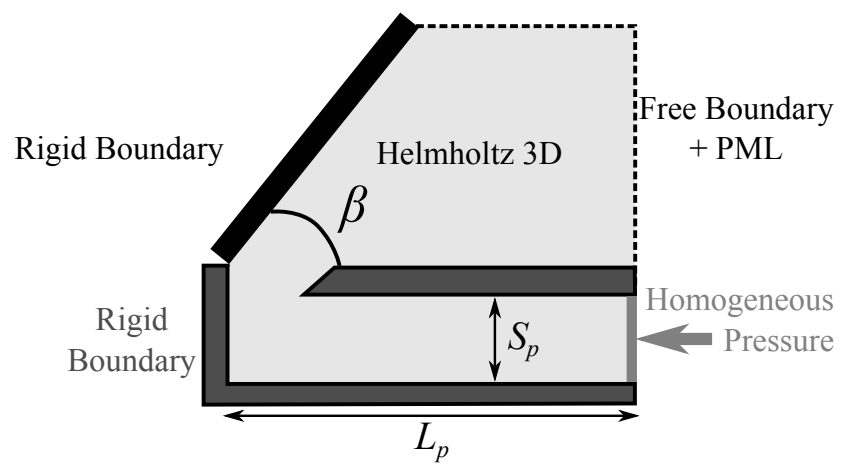

Figure 10: Sketch of the transverse section of the simulated geometry.

The mesh and the analysis of the simulations used in this study are adapted from a previous study [4] (Fig. 10). The mesh includes a rectangular tube with cross-sectional area $S_{p}$ and length $L_{p}$, a radiation domain limited by the inclined plane, and the opening. The geometry of the opening is characterized by two areas due to the shape of the edge: an internal area $S_{i}$ and an external area $S_{o}$ (Fig. 4). The relation between these two areas is determined by the sharpness of the edge and the height of the wall. Assuming that for low frequencies $(\lambda$ larger than the cross-sectional dimensions), the sound propagation is influenced only by the magnitude of the cross-sectional areas $\left(S_{p}, S_{o}, S_{i}\right)$, but not their shape, so all areas are modeled as rectangular which simplifies the generation and the configuration of the mesh.

For each given geometry, the Helmholtz equation (Eq.(20)), assuming a frictionless adiabatic flow, is solved for different values of the wave-number $k$ :

$$
\Delta P_{a c}(k)+k^{2} P_{a c}(k)=0
$$

where $P_{a c}(k)$ is the amplitude of the acoustic pressure for a given wavenumber. A homogeneous pressure $P_{a c}=p_{a c}^{0}$ is applied across the end of the tube (Fig. 10). Rigid boundary conditions are imposed on the wall and on the inclined plane, i.e. a zero normal acoustic velocity: $\frac{\partial P_{a c}}{\partial n}=0$, with $n$ the normal direction of the wall. To limit the influence of the boundary of the calculated radiation domain, artificial damping is added at the 
limit of the radiation domain, which obeys the Perfect Matched Layer condition (PML, $\frac{\partial P_{a c}}{\partial n}=-i k(1+i \nu) P_{a c}$, with $\nu=1$ controlling the damping) [27, 28].

The tube and the radiation domain are taken to be great enough for it to be assumed that the conditions at the boundaries of the simulated domain don't influence the response of the window. With these boundary conditions and the size of the mesh, the inclined plane behaves as an infinite plane. The length of the tube is chosen such that the wave propagation can be assumed to be planar at its end $\left(L_{p}=0.3 \lambda\right.$ with $\lambda$ the wave length, Fig. 10). The length is therefore modified for each value of $k$. The mesh has to be detailed enough to describe correctly the propagation through the opening. These two conditions limit the frequency domain which can be studied using a reasonable number of elements and computational cost. The lowest frequency is fixed to $f=100 \mathrm{~Hz}$, the same used for the measurements. The highest frequency is selected to match the cutoff frequency of the tube: $f<\frac{2 c}{h_{p}}$, with $h_{p}$ the longest dimension of the tube cross-section.

As with the measurements, the radiation impedance can not be obtained directly from the simulation, but has to be evaluated from the input impedance at the end of the tube. This input impedance is calculated from the pressure field predicted by the solution of the Helmholtz equation (20). It is defined as the ratio of the homogeneous pressure $p_{a c}^{0}$ over the mean normal velocity at the entrance of the pipe:

$$
Z_{i n}=\frac{p_{a c}^{0} S_{p}}{\int_{S_{p}^{0}} \mathbf{V}_{\mathbf{a c}} \mathbf{d} \mathbf{S}}=i k \rho c \frac{p_{a c}^{0} S_{p}}{\int_{S_{p}^{0}} \frac{d P_{a c}}{d x} d S},
$$

where the superscript 0 specifies that the integration is calculated at the entrance of the pipe. The acoustic velocity $V_{a c}$ is obtained from the spatial derivative of the acoustic pressure $P_{a c}$. The radiation impedance is obtained from this input impedance using a formula similar to Eq.(10). Following the frictionless and adiabatic assumption of Eq. (20), the visco-thermal losses are not taken into account in the simulation, so $\Gamma=i k$ and $Z_{c}=\rho c / S_{p}\left(f_{\Gamma}=f_{Z_{c}}=1\right)$. The radiation impedance $Z_{r}$ is written as:

$$
Z_{r}=\frac{\left.Z_{c} \tanh \left(i k L_{p}\right)-Z_{i n}\right)}{\frac{Z_{i n}}{Z_{c}} \tanh \left(i k L_{p}\right)-1} .
$$

The boundary condition assumptions and the method to obtain the radiation impedance are first tested on a well documented simple geometry: an unflanged open cylinder. After validation by comparison with the analytical solution, this method is tested on a geometry equivalent to the one measured. To ensure this equivalence, the mesh is generated using the dimensions of the experimental setup: the cross section of the pipe $S_{p}$, the internal cross section of the opening $S_{i}$ and the outside cross section $S_{o}$. The tilt angle of the plane is varied in the range $\pi / 36<\beta<\pi$. In the simulation, the propagation into the pipe is modeled under ideal circumstances: there are no visco-thermal losses and the dimensions of the pipe are precisely known $\left(L_{p}\right.$ and $\left.S_{p}\right)$. The radiation impedance $Z_{r}$ is then estimated by Eq. (22) with much better accuracy than when using the measurements. It provides smoother imaginary and real parts (Fig. 5, dashed lines).

The simulated imaginary components correspond well with the measured ones, especially at low frequencies, except for the smallest angle $\beta=\pi / 36$ (Fig. 5). For this configuration, the simulated radiation domain is small due to the inclined plane. The intensity of the acoustic pressure at the PML boundary condition is therefore high, which adversely affects the resulting simulation. For this angle, the simulations are not valid and are excluded from the analysis. For the other angles, the normalized imaginary part is constant at low frequencies. Inertances are therefore estimated from these simulations by taking the average of the normalized imaginary part in the same frequency range as before $\left(k r_{p} \in[0.05,0.25]\right)$.

The radiation in the absence of a plane $m_{r 0}$ is simulated with a geometry similar that of a plane with $\beta=\pi$ (Fig. 10) and by changing the boundary condition on the "plane" to a radiating condition (PML). The obtained values for $m_{r 0}$ are consistent with the prediction of the model used in a previous study [4] accounting for the opening geometry.

After removing the inertance $m_{r 0}$, a model similar to the one proposed for the measurements is fitted to the values of the plane inertances $m_{\beta}$ (Eq.(16)). Both the inertances and the coefficient $C$ obtained by simulation match those derived from measurements (Fig. 7) $)^{2}$. The relative difference between the estimation of the fitted model and the simulated data remains under $5 \%$ which again validates the choice of the model (Fig. 8).

These observations indicate that the effect of the inclined plane on the imaginary part of the radiation impedance is similar for the numerical simulations and the measurements. It validates numerical simulation as an efficient tool to study how the effect of the plane is influenced by the geometry of the aperture.

\footnotetext{
${ }^{2}$ For the F.E., the bias $\epsilon_{0}$ is of the order of magnitude as the standard deviation of the normalized imaginary component around its mean value $\left(4 \%\right.$ of $\left.m_{r 0}\right)$.
} 


\subsection{Imaginary component}

Simulations are run for 31 different geometries of the opening for which the inside cross sectional area varies over the range $0.2 \mathrm{~cm}^{2}<S_{i}<1.6 \mathrm{~cm}^{2}$ and the outside cross sectional area varies independently over the range $0.2 \mathrm{~cm}^{2}<S_{o}<2.5 \mathrm{~cm}^{2}$, with $S_{i} \leq S_{o}$ (Fig. 4). The cross sectional area of the pipe is kept constant $S_{p}=$ $1.2 \mathrm{~cm}^{2}$. For each of these opening geometries the radiation is simulated for different positions of the inclined plane $(\pi / 18 \leq \beta \leq \pi)$. For each opening geometry, the model proposed by Eq. (16) is fitted resulting in one value of the coefficient $C$ per opening geometry.

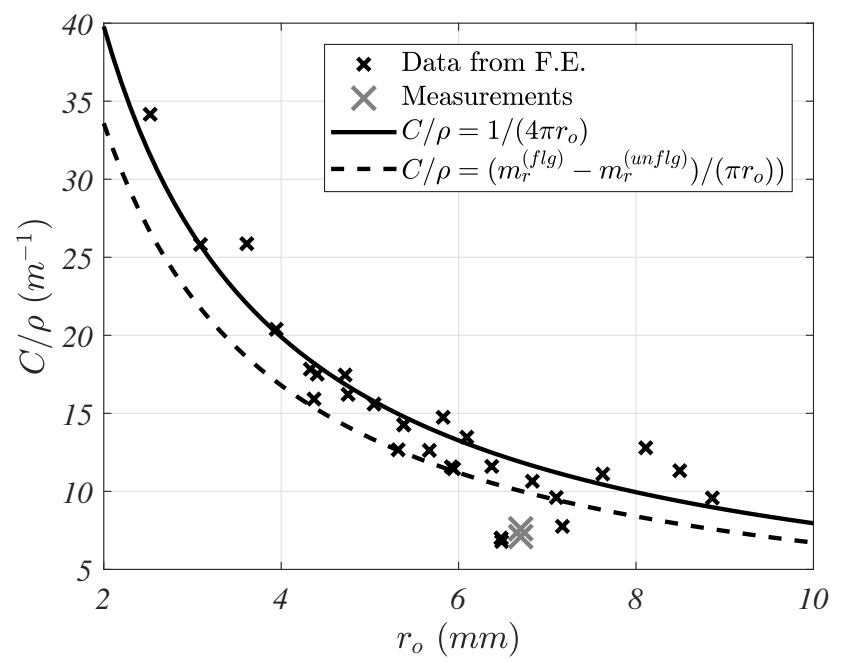

Figure 11: Evolution of the fitted parameter $C$ with the geometry of the opening, with the radius $r_{o}$ corresponding to the external section of the opening.

By following the relation between the radiation inertance and the equivalent radius of the opening area established for the unflanged and the flanged pipes (Eq.(3) and Eq.(4)), the evolution of the coefficient $C$ with the inverse of this radius $r_{o}=\sqrt{S_{o} / \pi}$ is observed (Fig. 11). These two parameters appear to be approximately proportional:

$$
C \approx \frac{\rho}{4 \pi r_{o}} .
$$

This relation appears to be slightly dependent on the exact geometry of the opening (internal area $S_{i}$, sharpness of the edge, etc.) which explains the variation of $C$ for similar values of the radius $r_{o}$ (Fig. 11). Finally, the radiation inertance $m_{r}$ is predicted by the following expression:

$$
m_{r}=m_{\beta}+m_{r 0}=\frac{\rho}{4 \pi r_{o}} \cot \left(\frac{\beta}{4}\right)+m_{r 0} .
$$

This formulation is consistent with the radiation inertances of the well established analytical solutions for the unflanged and flanged pipe. Indeed, taking into account that the radiation domain is delimited by the pipe and the inclined plane, the radiation should be similar to that of a flanged pipe if $\beta=\pi$ and similar to that of an unflanged pipe if $\beta=2 \pi$. The proposed model (Eq.(24)) gives for the unflanged pipe:

$$
m_{r}^{(\text {unflg })}=m_{r}(\beta=2 \pi)=m_{r 0},
$$

and for the flanged pipe:

$$
\begin{aligned}
m_{r}^{(f l g)}=m_{r}(\beta=\pi) & =\frac{\rho}{4 \pi r_{o}} \cot \left(\frac{\pi}{4}\right)+m_{r 0} \\
& =\frac{\rho}{\pi r_{o}} 0.25+m_{r 0} .
\end{aligned}
$$

The difference between these two inertances is:

$$
\left(m_{r}^{(f l g)}-m_{r}^{(\text {unflg })}\right)=\frac{\rho}{\pi r_{o}} 0.25 \approx \frac{\rho}{\pi r_{o}}(0.824-0.613),
$$

which is consistent with the established value (Eq.(3),(4)). Conversely, consistency with the established value can be imposed by fixing $C=\left(m_{r}^{(f l g)}-m_{r}^{(\text {unflg })}\right) \rho /\left(\pi r_{o}\right)$. This expression is a good approximation of the simulated data (Fig. 11). 
This model can be applied to the case of the notch flute used in this study. The prediction is compared to the measurement in Figure 7. For this geometry of opening, the model proposed here overestimates the value of the coefficient $C$ (measured: $C \approx 7$, predicted $C \approx 10$ ). Indeed, the results of the fit are slightly lower than the linear regression (Fig. 11).

Furthermore, the obtained expression (Eq.(24)) differs only by a factor of $1 / 2$ from the main term of the inertance of a truncated cone of angle $\beta$ (Eq.(7) and Eq.(8), with $\theta=\beta / 2$ ). In particular, the two models have the same dependence with the angle in $\cot (\beta / 4)$ (Eq.(7) and Eq.(24)).

The inertance correction $m_{\beta}$ induced by the plane is inversely proportional to $r_{o} \tan (\beta / 4)$ (see Eq.(24)). It can be interpreted as an equivalent distance between the inclined plane and the opening. This observation is therefore consistent with the effect of a parallel plane hanging above a hole [18] (Sec. 1.2).

\subsection{Real component}

The simulated real parts are smoother than the measured real components (Fig. 5,b) due to the absence of unknowns in the model of the propagation into the pipe used in Eq. (22) (Sec. 4.1). They can therefore be used to estimate the coefficient $\zeta$ which links the real part to the square of the wavenumber $k$ (Eq.2). The value of the coefficient $\zeta$ is estimated for each tilt angle $\beta$ and for each opening geometry by taking the average of the normalized real part of the radiation impedance in the frequency range $k r_{n} \in[0.05,0.5]$..
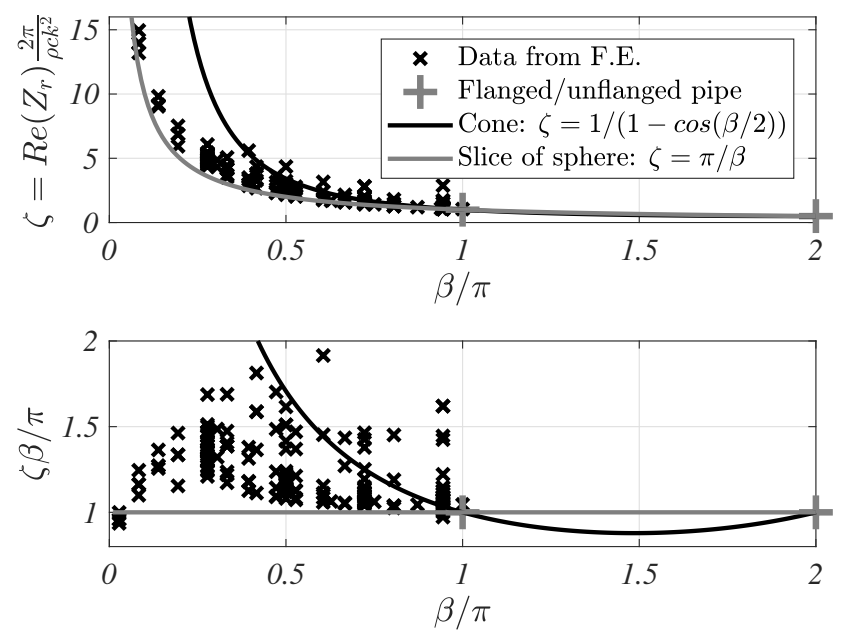

Figure 12: Evolution of the coefficient $\zeta$ characterizing the real part of the radiation impedance with the angle $\beta$ of the plane $(a)$. Its multiplication by $\beta / \pi$ enhances the asymptotic behavior of $\zeta$ for small angle $(b)$.

The coefficient $\zeta$ appears to be much more sensitive to the angle of the plane $\beta$ than to the opening geometry (Fig. 12,a). This observation is consistent with the formulation existing for the unflanged and flanged pipe in which the real part is independent of the opening size (Eq.(3),Eq.(4)). To a first approximation, the coefficient $\zeta$ can considered independent of the opening geometry.

The real part of the radiation impedance can be exactly calculated by ensuring the conservation of both the acoustic power and the flow rate between the output of the hole and a wavefront far away from the hole [24] (chap.12.6.1,p.681-683). If the wavefront is a portion of sphere of radius $R$ then the real part of the radiation impedance is [24] (chap.12.6.1,p.681-683):

$$
\Re e\left(Z_{r}\right)=\frac{\rho c}{S_{\text {rad }}} \frac{k^{2} R^{2}}{1+k^{2} R^{2}}
$$

where $S_{\text {rad }}$ is the surface area of the wavefront.

a)

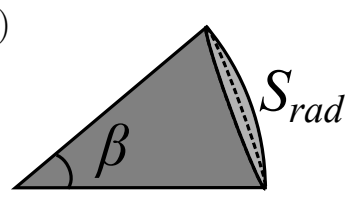

b)

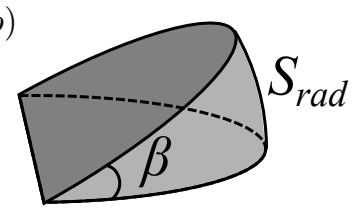

Figure 13: Sketch of radiating domain: cone $(a)$ and slice of sphere $(b)$. 
For the geometry studied here, the radiation domain can be assumed to be limited by the inclined plane and the pipe. The geometry of the resulting radiation domain is not obvious. If this domain is a cone of angle $\beta$, the area of the wavefront is $S_{\text {rad }}^{\text {cone }}=2 \pi R^{2}(1-\cos (\beta / 2))$ (Fig. 13, a). At low frequencies, it is shown that $[21,24]$ (chap.12.6.1,p.681-683):

$$
\Re e\left(Z_{r}^{\text {cone }}\right)=\frac{\rho c k^{2}}{2 \pi(1-\cos (\beta / 2))}
$$

The coefficient $\zeta^{(\text {cone })}=1 /(1-\cos (\beta / 2))$ is similar to that of a wide truncated cone Eq.(8) (Sec. 1.2). If the radiation domain is a slice of sphere of angle $\beta$ (Fig. $13, b)$, the radiation surface is: $S_{\text {rad }}^{\text {slice }}=2 R^{2} \beta$. For low frequencies, this leads to:

$$
\Re e\left(Z_{r}^{\text {slice }}\right)=\frac{\rho c k^{2}}{2 \beta} .
$$

The coefficient characterizing the frequency dependence of the real part is therefore:

$$
\zeta=\frac{\pi}{\beta} .
$$

These expressions are compared to the coefficient estimated from the FE simulations (Fig. 12, b). The coefficient $\zeta$ multiplied by the angle $\beta$ does not diverge for small angles, which supports the suggestion that the radiation domain should be described as a slice of a sphere rather than as a cone.

For $\beta=\pi$, the radiation domain is a hemisphere, which is similar to that of a flanged pipe and $\zeta(\beta=\pi)=$ $\zeta^{(f l g d)}=1$. For $\beta=2 \pi$, the radiation domain is a full sphere, which corresponds to an unflanged pipe and $\zeta(\beta=2 \pi)=\zeta^{(\text {unflgd })}=1 / 2$ (Fig. 13, b). The proposed equation (31) is therefore consistent with these two well established cases.

\section{Conclusions}

With the goal of better understanding the influence of the musician's face position on the acoustic response of a flute-like instrument, this work considers a simplification of the problem: the influence of an inclined plane on the radiation of a hole. This study proposes a model for both the imaginary and real components of the radiation impedance based on measurements and numerical simulations. The size of the plane does not appear to influence the imaginary part of the radiation impedance provided that the plane is larger than the opening $\left(S_{\text {plane }}>S_{o}\right)$ (Sec. 2.3). The influence of the tilt angle $\beta$ of the plane on the radiation impedance $Z_{r}$ is well modeled by the following expression:

$$
Z_{r}=j\left(\frac{\rho \cot (\beta / 4)}{4 \pi r_{o}}+m_{r 0}\right) c k+\frac{\pi}{\beta} \frac{\rho c}{2 \pi} k^{2},
$$

with $r_{o}$ the radius corresponding to the external area of the hole $\left(r_{o}=\sqrt{S_{o} / \pi}\right), m_{r 0}$ the inertance of the hole without a plane, $k$ the wavenumber, $\rho$ the air density and $c$ the speed of sound ${ }^{3}$. Substituting the characteristic impedance $Z_{c}=\rho c / S_{o}$, expression Eq.(32) becomes:

$$
Z_{r}=Z_{c}\left[j\left(\frac{\cot (\beta / 4)}{4} r_{o}+\frac{S_{o}}{\rho} m_{r 0}\right) k+\frac{\pi}{2 \beta}\left(k r_{o}\right)^{2}\right]
$$

This model is consistent with the well established radiation impedance of an unflanged and flanged pipe, by assuming that a flanged pipe corresponds to a plane inclined with an angle $\beta=\pi$, and that the unflanged pipe corresponds to the position of the plane $\beta=2 \pi$, for which it has no more effect on radiation (Sec. 2.4).

These formulations are established and validated only for low frequencies $f<1500 \mathrm{~Hz}$, corresponding to $k r_{p}<0.25$ or $k r_{o}<0.25$. For higher frequencies, the measurements diverge slightly from the low frequency behavior (Fig. 5), which is not observed in the F.E. simulation. This phenomenon is possibly due to inaccuracies of data processing methods, such as the value of the parameters of the sound propagation model used to estimate the radiation impedance (eq.10 and eq.12) (Sec. 2.1). According to the numerical simulations, the frequency range for which the proposed model is valid could be extended to $k r_{p}<0.5$ (Fig. 5 ).

In this study, the modification of the radiation by the musician's face is separated into two effects: the reduction of the opening area and the presence of an inclined plane in front of the opening (Sec. 1). The

\footnotetext{
${ }^{3}$ The bias $\epsilon_{0}$ introduced in Eq.(17) and discussed in sections 2.4 and 3.2 is here neglected.
} 
proposed expression (Eq. 32) can model these two effects, observing that the inertance $m_{r 0}$ is also modified by the reduction of the opening area $[17,4]$.

In the current work, only the radiation impedance $Z_{r}$ is modeled. To obtain the complete impedance of a side hole or window, additional elements must be added to account for the geometry of the opening [16, 17, 4]: the change in the direction of the propagation, the change of cross-sectional area, the height of the wall, the enlargement of the hole, etc.

This study also proposes a new method of measuring the variation of an inertance. This method is limited by some strong assumptions. For example, the independence of the inertance with the frequency can not be verified. This method can only be used to measure a modification of the imaginary component. However, the protocol benefits from a simple set-up and analysis, and can easily be adapted for instrument makers. Furthermore, it provides information regarding the influence of the imaginary part of the radiation impedance at standard playing level, where vortex shedding at the labium may appear [29].

\subsection{Future work}

This work is a first step in understanding the link between the positioning of the musician's face and the geometry of the flute-like instrument. The next step is to link this model to the actual position of a player, including how this varies during musical performances. In previous studies, the position of the player's face is reduced to the distance between the player's lips and the edge of the flute $[5,6,8,9,10]$. The relation between the distance and the geometric parameters used in this study (radius of the opening section $r_{o}$ and tilt angle $\beta$ ) is not obvious and should be investigated. In these studies, the position of the lips is extracted from videos focused on the player's mouth. A similar setup could be used to observe the actual position of the player's face during a performance.

Once this is completed, it will be possible to estimate the acoustic resonances of the instrument in the presence of a musician. With the current knowledge of the source mechanism of this type of instrument [2, 30, 31] it is already possible to link the acoustic resonances to the sounding frequencies through the hydrodynamic parameters of the jet (size, velocity, etc.). Finally, with these different elements, it should be possible to link the geometry of the instrument to the necessary control gesture of the musician including both the face position and jet parameters. These results could be used to interpret the geometry of the instrument in terms of playability [13], or used in optimization algorithms.

\section{Acknowledgement}

The authors would like to thank V. Wetzel and O. Roulleau-Thery for their help with the preliminary step of the experimental part of the work presented here and J. Kergomard for the instructive discussions on the theoretical aspects of this study. This work is supported by CONICYT through FONDECYT Project No.1161464.

\section{A Appendix}

Assuming that the recorder is a cylinder with a length $L_{s}$ and a cross sectional area $S_{s}$ associated with a characteristic impedance $Z_{c s}$, the input impedance of the resonator seen by the sound source $Z_{t o t}$ is:

$$
Z_{t o t}=Z_{c s} \tanh \left[\Gamma_{s} L_{s}+\tanh ^{-1}\left(\frac{Z_{i n}}{Z_{c s}}\right)\right],
$$

with $Z_{\text {in }}$ the input impedance of the notch flute head. Assuming that this head is a cylinder of length $L_{p}$ and cross sectional area $S_{p}$ with a characteristic impedance $Z_{c p}$, Eq.(34) becomes:

$$
Z_{i n}=Z_{c p} \tanh \left[\Gamma_{p} L_{p}+\zeta(k R)^{2}\right]+j \omega m_{r}
$$

where $m_{r}$ is the radiation inertance. Assuming that this inertance is small compared with the modulus of the input impedance $Z_{i n}$, it is possible expand the inverse hyperbolic tangent in a Taylor series:

$$
\tanh ^{-1}\left(\frac{Z_{i n}}{Z_{c s}}\right) \approx \tanh ^{-1}(A)+\frac{j \omega}{Z_{c s}} m_{r} \frac{1}{1-A^{2}}
$$

with

$$
A=\frac{S_{p}}{S_{s}} \tanh \left[\Gamma_{p} L_{p}+\zeta(k R)^{2}\right]
$$


This expression can be simplified by neglecting the visco-thermal losses $\left(\Gamma_{p} \approx j k\right)$ and the real part which is small compare to the imaginary part in low frequency range $\left(k \ll L_{p} / R^{2}\right)$ :

$$
A \approx j\left(\frac{S_{p}}{S_{s}}\right) \tan \left(k L_{p}\right)
$$

The Taylor expansion of Eq. 36 can be used in Eq. 34 which results in the total input impedance:

$$
\begin{aligned}
Z_{t o t}=Z_{c s} \tanh & {\left[\Gamma_{s} L_{s}+j k \frac{S_{s}}{\rho} m_{r} \frac{1}{1-A^{2}}\right.} \\
& \left.+\tanh ^{-1}(A)\right] .
\end{aligned}
$$

The presence of the plane modifies the radiation inertance $m_{r}^{\prime}=m_{r}+m_{\beta}$ which has a similar effect on the total impedance $Z_{t o t}$ as a length correction $\Delta l_{e q}$ :

$$
\Delta l_{e q}=\frac{S_{s}}{\rho} m_{\beta} \frac{1}{1-A^{2}} .
$$

The link between the shift of the sounding frequency and the added inertance is finally:

$$
m_{\beta}=\frac{\rho}{S_{s}}\left(1-A^{2}\right)\left(\frac{c_{0}}{2 f}-\frac{c_{0}}{2 f_{0}}\right) .
$$

\section{References}

[1] B. Fabre and A. Hirschberg, "Physical modeling of flue instruments: a review of lumped models," Acta Acustica united with Acustica, vol. 86, no. 4, pp. 599-610, 2000.

[2] R. Auvray, B. Fabre, and P.-Y. Lagrée, "Regime change and oscillation thresholds in recorder-like instruments," The Journal of the Acoustical Society of America, vol. 131, pp. 1574-1585, Feb. 2012.

[3] M.-P. Verge, Aeroacoustics of confined jets : with applications to the physical modeling of recorder-like instruments. PhD thesis, Technische Universiteit Eindhoven, 1995.

[4] A. Ernoult and B. Fabre, "Window Impedance of Recorder-Like Instruments," Acta Acustica united with Acustica, vol. 103, pp. 106-116, Jan. 2017.

[5] J. W. Coltman, "Resonance and Sounding Frequencies of the Flute," The Journal of the Acoustical Society of America, vol. 40, pp. 99-107, July 1966.

[6] N. H. Fletcher, "Acoustical correlates of flute performance technique," The Journal of the Acoustical Society of America, vol. 57, no. 1, pp. 233-237, 1975.

[7] P. Dickens, Flute acoustics: measurement, modelling and design. PhD thesis, University of New South Wales, Nov. 2007.

[8] P. de la Cuadra, B. Fabre, N. Montgermont, and C. Chafe, "Analysis of Flute Control Parameters: A Comparison Between a Novice and an Experienced Flautist," Acta Acustica united with Acustica, vol. 94, pp. 740-749, Sept. 2008.

[9] I. Cossette, B. Fabre, V. Fréour, N. Montgermont, and P. Monaco, "From Breath to Sound: Linking Respiratory Mechanics to Aeroacoustic Sound Production in Flutes," Acta Acustica united with Acustica, vol. 96, pp. 654-667, July 2010.

[10] C. Vauthrin, B. Fabre, and I. Cossette, "How Does a Flute Player Adapt His Breathing and Playing to Musical Tasks?" Acta Acustica united with Acustica, vol. 101, pp. 224-237, Mar. 2015.

[11] J. Wolfe, J. Smith, J. Tann, and N. H. Fletcher, "Acoustic impedance spectra of classical and modern flutes," Journal of Sound and Vibration, vol. 243, pp. 127-144, May 2001.

[12] C. Balosso-bardin, P. De la Cuadra, C. Vauthrin, and B. Fabre, "An interdisciplinary study of the 1832 Boehm flute: history, geometry and acoustics," Musique et Technique, vol. 7, pp. 11-24, Mar. 2017.

[13] P. de la Cuadra, A. Ernoult, C. Balosso-Bardin, and B. Fabre, "Playability in flute-like instruments: Investigating the relation between flute making and instrumental control," The Journal of the Acoustical Society of America, vol. 141, pp. 3874-3874, May 2017.

[14] P. De La Cuadra, C. Balosso-Bardin, C. Vauthrin, and B. Fabre, "Optimized redesing of the quena," in EuroRegio, (Porto, Portugal), June 2016.

[15] H. Levine and J. Schwinger, "On the radiation of sound from an unflanged circular pipe," Physical review, vol. 73, no. 4 , p. $383,1948$. 
[16] Keefe, "Theory of the single woodwind tone hole," The Journal of the Acoustical Society of America, vol. 72, pp. 676-687, Sept. 1982.

[17] V. Dubos, J. Kergomard, A. Khettabi, J.-P. Dalmont, D. H. Keefe, and C. J. Nederveen, "Theory of sound propagation in a duct with a branched tube using modal decomposition," Acta Acustica united with Acustica, vol. 85, no. 2, pp. 153-169, 1999.

[18] J.-P. Dalmont, C. Nederveen, and N. Joly, "Radiation impedance of tubes with different flanges: numerical and experimental investigations," Journal of Sound and Vibration, vol. 244, pp. 505-534, July 2001.

[19] J.-P. Dalmont, C. J. Nederveen, V. Dubos, S. Ollivier, V. Méserette, and E. te Sligte, "Experimental Determination of the Equivalent Circuit of an Open Side Hole: Linear and Non Linear Behaviour," Acta Acustica united with Acustica, vol. 88, pp. 567-575, July 2002.

[20] F. Silva, P. Guillemain, J. Kergomard, B. Mallaroni, and A. N. Norris, "Approximation formulae for the acoustic radiation impedance of a cylindrical pipe," Journal of Sound and Vibration, vol. 322, pp. 255-263, Apr. 2009.

[21] J. Kergomard, A. Lefebvre, and G. P. Scavone, "Matching of Fundamental Modes at a Junction of a Cylinder and a Truncated Cone; Application to the Calculation of Some Radiation Impedances," Acta Acustica united with Acustica, vol. 101, pp. 1189-1198, Nov. 2015.

[22] J. C. Le Roux, M. Pachebat, and J.-P. Dalmont, "A new impedance sensor for industrial applications," in Acoustics 2012 (S. F. d'Acoustique, ed.), (Nantes, France), p. ., Apr. 2012.

[23] A. Lefebvre, Computational acoustic methods for the design of woodwind instruments. PhD thesis, McGill University, 2010.

[24] A. Chaigne and J. Kergomard, Acoustics of Musical Instruments. Modern Acoustics and Signal Processing, New York, NY: Springer New York, 2016.

[25] D. H. Keefe, "Acoustical wave propagation in cylindrical ducts: Transmission line parameter approximations for isothermal and nonisothermal boundary conditions," The Journal of the Acoustical Society of America, vol. 75, pp. 58-62, Jan. 1984.

[26] P. Rucz, F. Augusztinovicz, J. Angster, T. Preukschat, and A. Miklós, "Acoustic behavior of tuning slots of labial organ pipes," The Journal of the Acoustical Society of America, vol. 135, pp. 3056-3065, May 2014.

[27] J.-P. Berenger, "A perfectly matched layer for the absorption of electromagnetic waves," Journal of computational physics, vol. 114, no. 2, pp. 185-200, 1994.

[28] R. Auvray, B. Fabre, and P. Y. Lagrée, "Determination of 2d Quasi Incompressible Flow around a Recorder Labium: a Comparison between Different Methods," in Stockholm Music Acoustics Conference, (Stockholm, Sweden), 2013.

[29] B. Fabre, A. Hirschberg, and A. P. J. Wijnands, "Vortex Shedding in Steady Oscillation of a Flue Organ Pipe," Acta Acustica united with Acustica, vol. 82, pp. 863-877, Nov. 1996.

[30] S. Terrien, C. Vergez, and B. Fabre, "Flute-like musical instruments: A toy model investigated through numerical continuation," Journal of Sound and Vibration, vol. 332, pp. 3833-3848, July 2013.

[31] R. Auvray, A. Ernoult, B. Fabre, and P.-Y. Lagrée, "Time-domain simulation of flute-like instruments: Comparison of jet-drive and discrete-vortex models," The Journal of the Acoustical Society of America, vol. 136, no. 1, pp. 389-400, 2014. 